\title{
SUVmax Predicts Disease Progression after Stereotactic Ablative Radiotherapy in Stage I Non-small Cell Lung Cancer
}

Yoo-Kang Kwak, MD, PhD'1
Hee Hyun Park, MD²
Kyu Hye Choi, MD2
Eun Young Park, MD2
Soo Yoon Sung, MD, PhD³
Sea-Won Lee, MD
Ji Hyun Hong, MD'
Hyo Chun Lee, MD
le Ryung Yoo, MD, PhD
Yeon Sil Kim, MD, PhD²

${ }^{1}$ Department of Radiation Oncology, Incheon St. Mary's Hospital, College of Medicine, The Catholic University of Korea, Seoul, ${ }^{2}$ Department of Radiation Oncology, Seoul St. Mary's Hospital, College of Medicine, The Catholic University of Korea, Seoul, ${ }^{3}$ Department of Radiation Oncology, Eunpyeong St. Mary's Hospital, College of Medicine, The Catholic University of Korea, Seoul, ${ }^{4}$ Department of Nuclear Medicine, Seoul St. Mary's Hospital, College of Medicine, The Catholic University of Korea, Seoul, Korea
Correspondence: Yeon Sil Kim, MD, PhD Department of Radiation Oncology, Seoul St. Mary's Hospital, College of Medicine, The Catholic University of Korea, 222 Banpo-daero, Seocho-gu, Seoul 06591, Korea Tel: 82-2-2258-6259

Fax: 82-2-2258-1532

E-mail: yeonkim7@catholic.ac.kr

Received January 2, 2019

Accepted May 16, 2019

Published Online May 17, 2019

\section{Purpose}

Fluorodeoxyglucose positron emission tomography-computed tomography (PET-CT) is gaining evidence as a predictive factor in non-small cell lung cancer (NSCLC). Stereotactic ablative radiotherapy (SABR) is the standard treatment in early-stage NSCLC when a patient is unsuitable for surgery. We performed a study to assess the prognostic clinical significance of PET-CT after SABR in early-stage NSCLC.

\section{Materials and Methods}

Seventy-six patients with stage I NSCLC treated with SABR were investigated. Total radiation dose ranged from 36 to 63 Gy in three to eight fractions depending on tumor location and size. Respiratory motion control was implemented at simulation and during treatment. PETCT prior to SABR was performed in 66 patients (86.8\%).

\section{Results}

Median follow-up time was 32 months (range, 5 to 142 months). Local control rate at 1, 2, and 5 years were $95.9 \%, 92.8 \%$, and $86.7 \%$, respectively. Overall survival (OS) at 1, 2, and 5 years were $91.0 \%, 71.3 \%$, and $52.1 \%$ respectively. Cause-specific survival at 1,2 , and 5 years were $98.6 \%, 93.1 \%$, and $84.3 \%$ respectively. Tumor size and pre-SABR maximal standardized uptake value (SUVmax) demonstrated statistical significance in the Kaplan-Meier survival analyses with log-rank test. In multivariate analyses pre-SABR SUVmax remained statistically significant in correlation to $\mathrm{OS}$ ( $p=0.024$; hazard ratio [HR], 3.2; 95\% confidence interval $[\mathrm{Cl}], 1.2$ to 8.8 ) and with marginal significance in regards to regional progressionfree survival ( $p=0.059 ; \mathrm{HR}, 32.5 ; 95 \% \mathrm{Cl}, 2.6$ to 402.5).

\section{Conclusion}

Pre-SABR SUVmax demonstrated a predictive power in statistical analyses. Tumors with SUVmax above 6 at diagnosis were associated with inferior outcomes.

\author{
Key words \\ Radiosurgery, Non-small-cell lung carcinoma, \\ Positron emission tomography computed tomography, \\ Prognostic factor
}




\section{Introduction}

Due to the advances in cancer screening and prolonged lifespan, diagnosis of early-stage non-small cell lung cancer (NSCLC) in high-risk patients is increasing. Sterotactic ablative radiotherapy (SABR) is a newer treatment option for these patients [1]. SABR is a promising new radiotherapy technology with an accurate delivery of high-dose radiation to a target. Moreover, it minimizes toxicity to its surrounding tissues. The treatment outcome is comparable to surgery, with a local control rate of approximately $90 \%$ at 3 years [2]. It is less invasive and can be delivered in an outpatient setting with a short treatment duration. The survival of the patients that receive SABR is continuously increasing because more fit patients are being treated with SABR [3]. However, since surgery is not operated, tissue specimen are not achieved and pathologic predictive factors cannot be assessed. In these circumstances, a predictor of outcomes after SABR in early NSCLC is in growing demand.

The recently presented 8th edition of the TNM Classification of Malignant Tumors by the American Joint Committee on Cancer recommends positron emission tomography-computed tomography (PET-CT) for initial work-up of patients [4]. Standardized uptake value (SUV), a measurement achieved through PET, is a relative measure of fluorodeoxyglucose (FDG) uptake. This is correlated with tumor doubling time and proliferation rates that reflect tumor aggressiveness. In a systematic review by the International Association for the Study of Lung Cancer (IASLC) Staging Project Group, PET SUV is considered prognostic in NSCLC in general $[5,6]$. Since most of the newly diagnosed NSCLC patients in available centers have baseline PET-CT taken and the maximal standard uptake value (SUVmax) is commonly reported, the baseline SUVmax might be a good candidate for the predictor of outcome after SABR for this early-stage NSCLC. Thus, we analyzed the association between baseline SUVmax and several outcome measures in order to identify its potential as a predictor of outcome in stage I NSCLC patients treated with SABR.

\section{Materials and Methods}

\section{Patient eligibility}

This study included stage I NSCLC patients that received SABR for lung lesions in a single institution between 2004 and 2016. Staging was performed according to the 7th edition of the American Joint Committee on Cancer Staging Manual
[7]. Inclusion criteria were follows: (1) non-small cell lung carcinoma; (2) tumors less than $5 \mathrm{~cm}$ in the greatest dimension; and (3) treated with SABR with a definitive aim. Exclusion criteria were follows: (1) positive regional lymph nodes and distant metastases confirmed by computed tomography (CT), PET-CT, or endobronchial ultrasound (EBUS); (2) patients treated on synchronous and/or metasynchronous metastatic lesions. Indications for SABR were mostly medically inoperable cases and other cases where surgery was refused. The medically inoperable cases included poor pulmonary function, end-organ damage, severe cerebral and / or cardiovascular disease, and chronic heart disease contraindicated to surgery. Among the 151 patients who were treated with SABR for lung lesions, the patients that were treated on metastatic lesions or treated with palliative aim were excluded. Finally, 76 patients were included. Some of the included patients were previously evaluated in another study [8].

\section{Evaluation}

For the diagnosis and clinical work-up, history taking and physical examination were done. The Eastern Cooperative Oncology Group (ECOG) criteria were used to assess the performance status. Routine complete blood counts $(\mathrm{CBC})$ and blood chemistry $(\mathrm{BC})$ were checked. To determine operability, patients were assessed by a thoracic surgeon or pulmonologist. Pulmonary function tests with carbon monoxide diffusion capacity and echocardiography were performed. Imaging studies for initial staging included chest $\mathrm{CT}$ with enhancement, PET-CT, bone scan, and brain magnetic resonance imaging (MRI).

\section{PET-CT procedure and analysis}

Patients were intravenously injected FDG with 5.5-7.4 $\mathrm{MBq} / \mathrm{kg}$ dosage after at least 6 hours of fasting. The PET-CT scanner Biograph Duo (Siemens Medical Solutions Inc., Knoxville, TN) was used for the acquisition of studies 60 minutes after injection, when the blood glucose level was maintained at $130 \mathrm{mg} / \mathrm{mL}$ and less. A non-enhanced CT scan with 5-mm slice thickness was performed initially from the orbitomeatal line to the proximal thigh. The same body region was scanned with PET immediately after.

After the attenuation correction with the obtained CT data, reconstruction using a standard ordered-subset expectation maximization algorithm was done. The images were reviewed by two nuclear medicine physicians. The SUVmax was evaluated by placing a volume of interest on the FDG-avid lesion using the Mirada XD3 software (Mirada Medical, Oxford, UK). The cut-off value of SUV 2.5 was used to define the contouring margin of the lesion. Each lesion was segmented in axial, sagittal, and coronal planes semi-automati- 
Table 1. Dose fractionation scheme for SBRT according to patient characteristics

\begin{tabular}{lcccl} 
Total dose (Gy) & $\begin{array}{c}\text { No. of } \\
\text { fractions }\end{array}$ & $\begin{array}{c}\text { Fraction } \\
\text { size (Gy) }\end{array}$ & $\begin{array}{c}\text { No. of } \\
\text { patients }(\%)\end{array}$ & Characteristic \\
\hline $36-48$ & 3 & $12-16$ & $9(11.8)$ & Relatively big tumor $(>4 \mathrm{~cm})$ \\
$48-60$ & $4-5$ & 12 & $54(71.1)$ & Peripheral tumor \\
$50-60$ & 6 & 10 & $6(7.9)$ & Central tumor \\
$56-63$ & $7-8$ & $7-8$ & $7(9.2)$ & High-risk central tumor $^{\text {a) }}$ \\
\hline
\end{tabular}

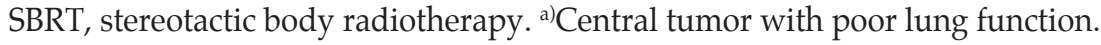

cally. SUVmax was calculated according to the final metabolic tumor volume quantified by the software.

\section{Treatment}

Three different radiotherapy modalities were used; cyberknife, helical tomotherapy, and static intensity-modulated radiotherapy (IMRT) with CT on-rail. Radiotherapy simulation was done in a supine position with both arms up together. Whole-body Vac-Lok (CIVCO Medical Solutions, Coralville, IW) was used for immobilization. When cyberknife was selected for treatment, fiducial markers were inserted prior to the simulation. Two to five fiducial markers (2-mm gold-pin markers) were implanted inside or near the target lesion through the use of a percutaneous needle by a specialized radiologist. For respiratory motion management, an abdominal compression with forced shallow breathing was used. Four-dimensional (4-D) CT scans with 2-mm sliced thickness were routinely performed for accurate target volumes. Tumor motion related to respiration was quantified by using 4-D CT scans.

The gross tumor volume (GTV) was delineated on every phase (5 inspiratory, 5 expiratory, and 1 resting phases), acquired by 4 -D CT scans on the pulmonary CT windows. The 11 GTVs acquired at each respiratory phase were integrated to generate internal target volume (ITV). To compensate for the intra- and inter-fractional variations in the patient position and beam geometries, 5-mm margins were added to the ITV to define the planning target volume. At each treatment, an image guidance to confirm the position of the target was achieved.

Radiation dose was delivered in three to eight fractions with a total dose of 36 to $63 \mathrm{~Gy}$. Main factors for deciding on dose scheme were tumor location (central vs. peripheral, or proximity to chest wall) and tumor size. The most commonly prescribed dose schedule was 60 Gy in five fractions for peripheral tumors and $50 \mathrm{~Gy}$ in five fractions for central tumors. We summarized the prescribed dose schedule in Table 1.

\section{Follow-up}

The first follow-up was conducted at 1-month post-radiotherapy. In addition to physical examination and blood work-up, including $\mathrm{CBC}$ and $\mathrm{BC}$, chest $\mathrm{X}$-ray, and chest $\mathrm{CT}$, were performed. The treatment response was evaluated using the Response Evaluation Criteria in Solid Tumors ver. 1.1. The patients were followed up every 3 months for the first 2 years and every 6 months for up to 5 years since diagnosis. Brain MRI was scanned every year or at the appearance of neurologic symptoms.

\section{Statistical analyses}

The primary endpoint of this study was to identify the factors predictive of outcomes in early-stage NSCLC treated with SABR. The outcome definitions were as follows: local control was defined as absence of progressive soft-tissue CT density and size increment in the treated site; regional progression was defined as a newly developed recurrent regional lymph node; distant progression was defined as a newly developed tumor at distant sites; overall survival was defined as the date of SABR initiation to the date of death by any cause or last follow-up; cause-specific survival was defined as the date of SABR initiation to the date of death by cancer-related events or last follow-up.

Chi-square or Fisher exact test was used to compare the incidences of categorical variables and Student's t test was used for continuous variables. Pearson's correlation analysis was performed to analyze on association between continuous variables. All survival outcomes were calculated using the Kaplan-Meier method with log-rank test. Cox proportional hazards regression model was used for univariate and multivariate analysis to analyze on the predictive factors for each survival outcomes (local control, regional and distant progression-free survival, and overall survival). The SUVmax cut-off value was determined by receiver operating characteristic (ROC) curve analysis and Cox proportional hazards model regarding each survival outcomes. All statistical tests with $p$-values less than 0.05 were considered to be 
Table 2. Patient, tumor, and treatment characteristics

\begin{tabular}{|c|c|}
\hline & $\begin{array}{l}\text { No. of patients }(\%) \\
\qquad(\mathrm{n}=76)\end{array}$ \\
\hline Age, median (range, yr) & $74.5(48-90)$ \\
\hline$\leq 70$ & $38(50.0)$ \\
\hline$>70$ & $38(50.0)$ \\
\hline \multicolumn{2}{|l|}{ Sex } \\
\hline Male & $61(80.3)$ \\
\hline Female & $15(19.7)$ \\
\hline \multicolumn{2}{|l|}{ ECOG PS } \\
\hline 0 & $8(10.5)$ \\
\hline 1 & $55(72.4)$ \\
\hline 2 & $13(17.1)$ \\
\hline \multicolumn{2}{|l|}{ Comorbidity } \\
\hline No & $7(9.2)$ \\
\hline Yes & $69(90.8)$ \\
\hline \multicolumn{2}{|l|}{ Pathology } \\
\hline Squamous cell carcinoma & $25(32.9)$ \\
\hline Adenocarcinoma & $38(50.0)$ \\
\hline Non-small cell carcinoma, NOS & $4(5.3)$ \\
\hline Unproven & $9(11.8)$ \\
\hline \multicolumn{2}{|l|}{ Stage } \\
\hline T1a & $38(50.0)$ \\
\hline $\mathrm{T} 1 \mathrm{~b}$ & $19(25.0)$ \\
\hline T2a & $19(25.0)$ \\
\hline \multicolumn{2}{|l|}{ Tumor size $(\mathrm{cm})$} \\
\hline$\leq 3$ & $60(78.9)$ \\
\hline$>3$ & $16(21.1)$ \\
\hline \multicolumn{2}{|l|}{ Location (distance from bronchial tree) } \\
\hline Peripheral $(>2 \mathrm{~cm})$ & $60(78.9)$ \\
\hline Central $(\leq 2 \mathrm{~cm})$ & $16(21.1)$ \\
\hline \multicolumn{2}{|l|}{ Location (lobe) } \\
\hline Right upper & $22(28.9)$ \\
\hline Right middle & $5(6.6)$ \\
\hline Right lower & $11(14.5)$ \\
\hline Left upper & $25(32.9)$ \\
\hline Left lower & $13(17.1)$ \\
\hline Pretreatment SUVmax, median (range) & $6.7(0.5-22)$ \\
\hline$\leq 6$ & $29(43.9)$ \\
\hline$>6$ & $37(56.1)$ \\
\hline \multicolumn{2}{|l|}{ SABR modality } \\
\hline Cyberknife & $29(38.2)$ \\
\hline Tomotherapy & $14(18.4)$ \\
\hline Static IMRT (CT-on-rail), median (range) & $33(43.4)$ \\
\hline Dose $\left(\mathrm{BED}_{10}\right)$ & $116(80-150)$ \\
\hline$<120$ & $39(51.3)$ \\
\hline$\geq 120$ & $37(48.7)$ \\
\hline
\end{tabular}

(Continued)
Table 2. Continued

\begin{tabular}{lc} 
Reasons for SABR & $\begin{array}{c}\text { No patients }(\%) \\
(\mathrm{n}=76)\end{array}$ \\
\hline Medically inoperable & $63(82.9)$ \\
\hline Poor pulmonary function & $40(52.6)$ \\
\hline Poor cardiac function & $4(5.3)$ \\
\hline Other medical illness & $19(25.0)$ \\
\hline Refusal of surgery & $13(17.1)$ \\
\hline
\end{tabular}

ECOG PS, Eastern Cooperative Oncology Group performance status; NOS, not otherwise specified; SUVmax, maximal standardized uptake value; SABR, stereotactic ablative radiotherapy; IMRT, intensity-modulated radiotherapy; BED, biologically effective dose.

statistically significant. The SPSS software ver. 24 (IBM Corp., Armonk, NY) was used for statistical analyses.

\section{Ethical statement}

This study was approved by the Institutional Review Board of Seoul St. Mary's Hospital (IRB No. KC18RESE0561). The informed consent was waived by the Institutional Review Board, since this was a retrospective analysis.

\section{Results}

\section{Patient characteristics}

Table 2 describes the characteristics of the enrolled patients. Median age was 74.5 years old. About $80 \%$ of the patients were male and were in good performance status of ECOG 0 or 1 . Over $90 \%$ of the patients had comorbidity and $50 \%$ had adenocarcinoma. The median tumor size was 1.9 $\mathrm{cm}$ (range, 0.7 to $4.5 \mathrm{~cm}$ ) and $21.1 \%$ of the tumors were $>3 \mathrm{~cm}$. Tumor location was identified according to the distance from the bronchial tree. Peripheral tumors, which were defined as more than $2 \mathrm{~cm}$ afar from the bronchial tree, accounted for approximately $80 \%$. Right upper lobe tumors were most common. Static IMRT was most frequently used, accounting for $43.4 \%$. Median biologically effective dose with $\alpha / \beta$ ratio $10\left(\mathrm{BED}_{10}\right)$ was 116 and $48.7 \%$ of the patients received $\mathrm{BED}_{10} \geq 120$. The median pretreatment SUVmax was 6.95 (range, 0.5 to 22.0). The most common reason for patients to choose SABR was medically inoperable state due to poor pulmonary function. Nearly $17 \%$ of the patients refused surgery. 
Table 3. Correlation between SUVmax and outcomes using receiver operating characteristic curve

\begin{tabular}{lccc} 
& $\begin{array}{c}\text { Area under } \\
\text { the curve }\end{array}$ & $\begin{array}{c}\text { Range of SUVmax with } \\
\text { p-value }\end{array}$ & $\begin{array}{c}\text { maximal Youden's index } \\
\text { Local control }\end{array}$ \\
Regional and distant progression-free survival & 0.72 & 0.048 & $4.45-6.55$ \\
Overall survival & 0.69 & 0.016 & $5.90-6.65$ \\
\hline
\end{tabular}

SUVmax, maximal standardized uptake value.

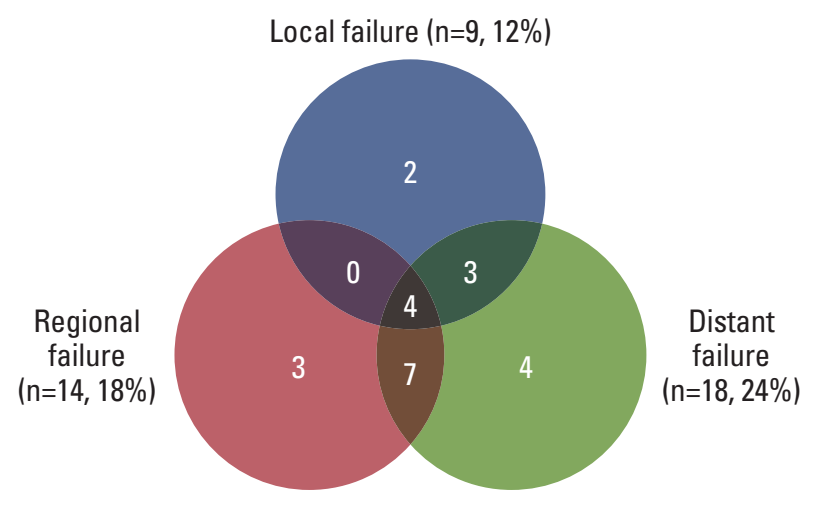

Fig. 1. Patterns of failure after stereotactic ablative radiotherapy.

\section{Determination of pretreatment SUVmax cut-off value}

ROC curve analysis was performed to determine the cutoff value for SUVmax (Table 3). The area under the curve was 0.72 and p-value was statistically significant with local control and recurrence-free survival. The range of SUVmax with Youden's J statistic index-a maximum value of (sensitivity+specificity-1)_calculated from the ROC curve is described in the table. We found a common value according to local control and recurrence-free survival, since overall survival and SUVmax did not show statistic correlation. We then designated 6 as the cut-off for analysis of the relationship between pretreatment SUVmax and outcome.

\section{Treatment outcomes}

Median follow-up time was 32 months (range, 5 to 142 months). Response to SABR was excellent with no progressive disease among the 71 evaluable patients. A complete response was observed in $32.4 \%(\mathrm{n}=23)$ and partial response in $43.7 \%(n=31)$. Seventeen patients $(23.9 \%)$ had stable disease. The patterns of failure are depicted in Fig. 1. Totally, 23 patients recurred and distant failure was the most common pattern of failure $(n=18)$, followed by regional failure $(n=14)$ and local failure $(n=9)$. Fourteen patients with distant recurrence also recurred locally and/or regionally. Two, three, and four patients had isolated local, regional, and distant recurrence, respectively.

One-, two-, and five-year local control rates were 95.9\%, $92.8 \%$, and $86.7 \%$, respectively. Two-year regional and distant progression-free survival rates were $86.0 \%$ and $82.3 \%$, respectively. Two-year overall survival rate was $69.8 \%$. Estimated lung cancer cause-specific survival rates at 1, 2, and 5 years were $98.6 \%, 93.1 \%$, and $84.3 \%$, respectively. The Kaplan-Meier curves for each survival outcomes above are depicted in Fig. 2A-E.

\section{Predictive factors for the treatment outcomes}

Results of univariate analyses of factors associated with local control, regional progression-free, distant progressionfree, and overall survival are summarized in Table 4 . The tumor size ( $\leq 3 \mathrm{~cm}$ vs. $>3 \mathrm{~cm}$ ) had a significant correlation with local control $(p=0.009)$ and overall survival $(p=0.042)$. The pretreatment SUVmax ( $\leq 6 \mathrm{vs.}>6$ ) showed a significant relationship with local control (100\% vs. $85.1 \%, \mathrm{p}=0.005)$, regional progression-free survival (96.6\% vs. 80.1\%, $\mathrm{p}=0.005)$, distant progression free survival (89.7\% vs. $78.4 \%, \mathrm{p}=0.038)$, and total progression-free survival (85.0\% vs. $66.0 \%, \mathrm{p}=0.029)$. Overall survival rates were numerically different in regards to pretreatment SUVmax ( $\leq 6 \mathrm{vs} .>6$ ), but the difference was not statistically significant $(86.9 \%$ vs. $60.5 \%, \mathrm{p}=0.088)$. Survival curves according to the pretreatment SUVmax are shown in Fig. 3.

Multivariate analyses of factors associated with local control, regional progression-free survival, distant progressionfree survival, and overall survival were also performed (Table 5). Pretreatment SUVmax was a predictive factor for overall survival $(\mathrm{p}=0.024)$, along with ECOG performance status $(p=0.001)$. Patients with SUVmax above 6 had a 3.2fold increase regarding death $(95 \%$ confidence interval [CI], 1.17 to 8.84). High pretreatment SUVmax showed decreased regional progression-free survival with borderline significance $(p=0.059)$ but was not significant in relation to local control and distant progression-free survival. Tumor size 
A
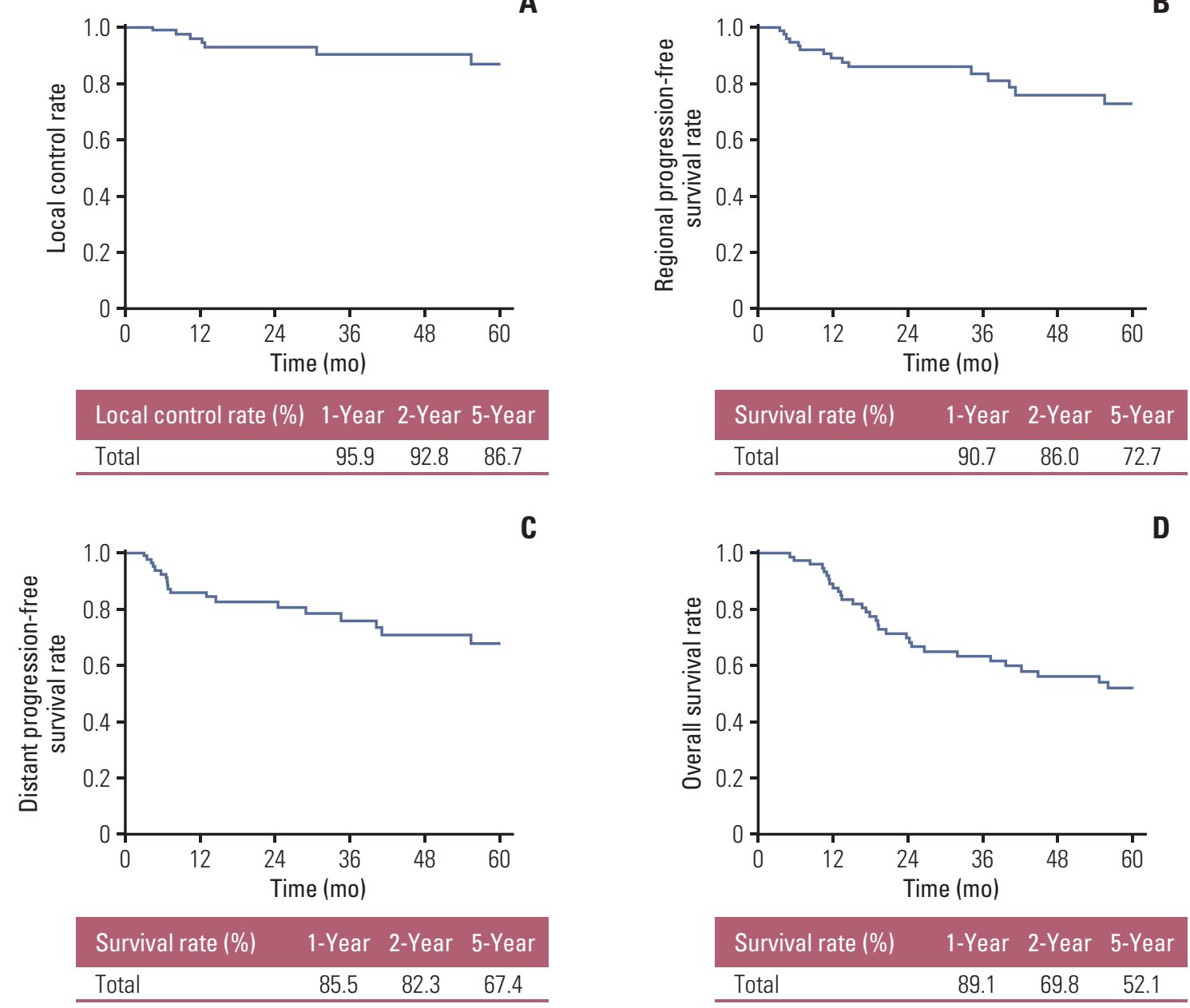

\begin{tabular}{lccc} 
Survival rate $(\%)$ & 1 -Year & 2 -Year & 5 -Year \\
Total & 89.1 & 69.8 & 52.1 \\
\hline
\end{tabular}

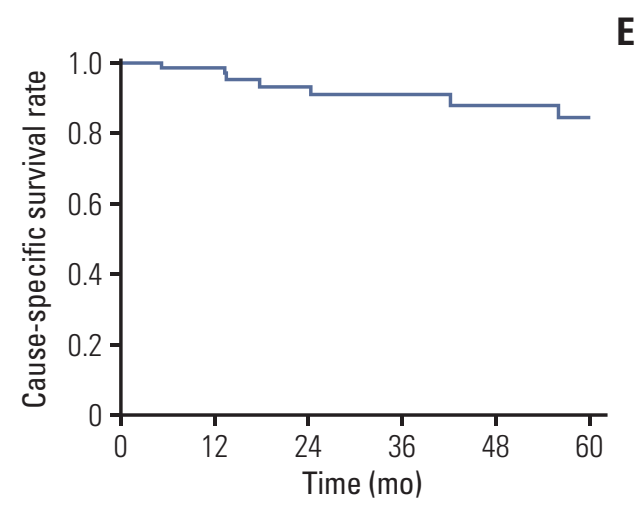

\begin{tabular}{lccc} 
Survival rate $(\%)$ & 1 -Year & 2 -Year & 5 -Year \\
\hline Total & 98.6 & 93.1 & 84.3 \\
\hline
\end{tabular}

Fig. 2. Treatment outcomes depicted in Kaplan-Meier survival curve. (A) Local control rate. (B) Regional progression-free survival rate. (C) Distant progression-free survival rate. (D) Overall survival rate. (E) Cause-specific survival rate. 
Table 4. Factors associated with LC, RPFS, DPFS, and OS on univariate analysis

\begin{tabular}{|c|c|c|c|c|c|c|c|c|}
\hline & \multicolumn{2}{|c|}{ LC } & \multicolumn{2}{|c|}{ RPFS } & \multicolumn{2}{|c|}{ DPFS } & \multicolumn{2}{|c|}{ OS } \\
\hline & $\begin{array}{c}\text { 2-Year } \\
\text { rate }(\%)\end{array}$ & p-value & $\begin{array}{c}\text { 2-Year } \\
\text { rate }(\%)\end{array}$ & p-value & $\begin{array}{c}2-Y e a r \\
\text { rate }(\%)\end{array}$ & p-value & $\begin{array}{c}\text { 2-Year } \\
\text { rate }(\%)\end{array}$ & p-value \\
\hline \multicolumn{9}{|l|}{ Age (yr) } \\
\hline$\leq 70$ & 100 & 0.154 & 79.0 & 0.833 & 79.5 & 0.956 & 78.3 & 0.224 \\
\hline$>70$ & 90.0 & & 88.9 & & 83.6 & & 66.6 & \\
\hline \multicolumn{9}{|l|}{ Sex } \\
\hline Male & 91.0 & 0.099 & 82.6 & 0.037 & 79.6 & 0.058 & 63.4 & 0.070 \\
\hline Female & 100 & & 100 & & 93.3 & & 100 & \\
\hline \multicolumn{9}{|l|}{ Pathology } \\
\hline Squamous cell carcinoma & 87.8 & 0.527 & 96.0 & 0.477 & 84.0 & 0.639 & 71.6 & 0.610 \\
\hline Adenocarcinoma & 94.0 & & 80.8 & & 78.6 & & 72.1 & \\
\hline \multicolumn{9}{|l|}{ Tumor size (cm) } \\
\hline$\leq 3$ & 96.1 & 0.009 & 89.2 & 0.062 & 85.7 & 0.111 & 77.3 & 0.042 \\
\hline$>3$ & 80.4 & & 74.5 & & 68.8 & & 53.3 & \\
\hline \multicolumn{9}{|l|}{ ECOG PS } \\
\hline 0 & 87.5 & 0.090 & 87.5 & 0.798 & 100 & 0.907 & 75.0 & 0.092 \\
\hline 1 & 92.2 & & 88.6 & & 81.4 & & 73.5 & \\
\hline 2 & 100 & & 75.2 & & 75.2 & & 51.9 & \\
\hline \multicolumn{9}{|l|}{ Comorbidity } \\
\hline No & 100 & 0.258 & 71.4 & 0.719 & 85.7 & 0.394 & 71.4 & 0.379 \\
\hline Yes & 92.0 & & 87.6 & & 81.9 & & 69.6 & \\
\hline \multicolumn{9}{|l|}{ Pretreatment SUVmax } \\
\hline$\leq 6$ & 100 & 0.005 & 96.6 & 0.005 & 89.7 & 0.038 & 86.9 & 0.088 \\
\hline$>6$ & 85.1 & & 80.1 & & 78.4 & & 60.5 & \\
\hline \multicolumn{9}{|l|}{ Dose $\left(\mathrm{BED}_{10}\right)$} \\
\hline$<120$ & 91.1 & 0.295 & 79.6 & 0.221 & 83.7 & 0.783 & 62.8 & 0.337 \\
\hline$\geq 120$ & 94.3 & & 91.9 & & 80.7 & & 80.1 & \\
\hline
\end{tabular}

LC, local control; RPFS, regional progression-free survival; DPFS, distant progression-free survival; OS, overall survival; ECOG PS, Eastern Cooperative Oncology Group performance status; SUVmax, maximal standardized uptake value; BED, biologically effective dose.

had no influence on treatment outcomes.

To assess on other characteristic differences according to SUVmax, we dichotomized the patients into two groups: SUVmax $\leq 6$ and SUVmax $>6$ (Table 6). Except for the tumor size, there was no difference in patient and tumor characteristics between the two groups. Only $8 \%$ of the patients in the SUVmax $\leq 6$ group had tumors greater than $3 \mathrm{~cm}$ while $29.4 \%$ of the patients accounted for tumors greater than $3 \mathrm{~cm}$ in the SUVmax $>6$ group $(\mathrm{p}=0.043)$.

\section{Discussion}

SABR has become a new standard in early-stage NSCLC for inoperable patients or those who refuse surgery. The local control rate of SABR reaches up to $90 \%$ at 3 years, which is comparable to surgery. Therefore, SABR is gaining evidence as a well-accepted strategy to substitute surgery for highrisk, early-stage lung cancer patients. Despite the superior treatment results, loco-regional and/or distant failure remains as an issue to be solved. Recognizing the factors predictive of recurrence after SABR in advance-at the time of treatment planning-can guide the physicians in deciding the treatment strategy and the aggressiveness of the therapy and follow up policy. To date, there are no proven predictive factors of NSCLC other than stage and performance status [9]. Other factors such as weight loss [10], tumor markers [11], molecular markers [12], and radiographic features detected in CT [13] have also been suggested as potential predictors. However, these require further validation.

PET-CT is a useful tool not only for systemic staging but also for the decision of treatment approach and extent. 
A
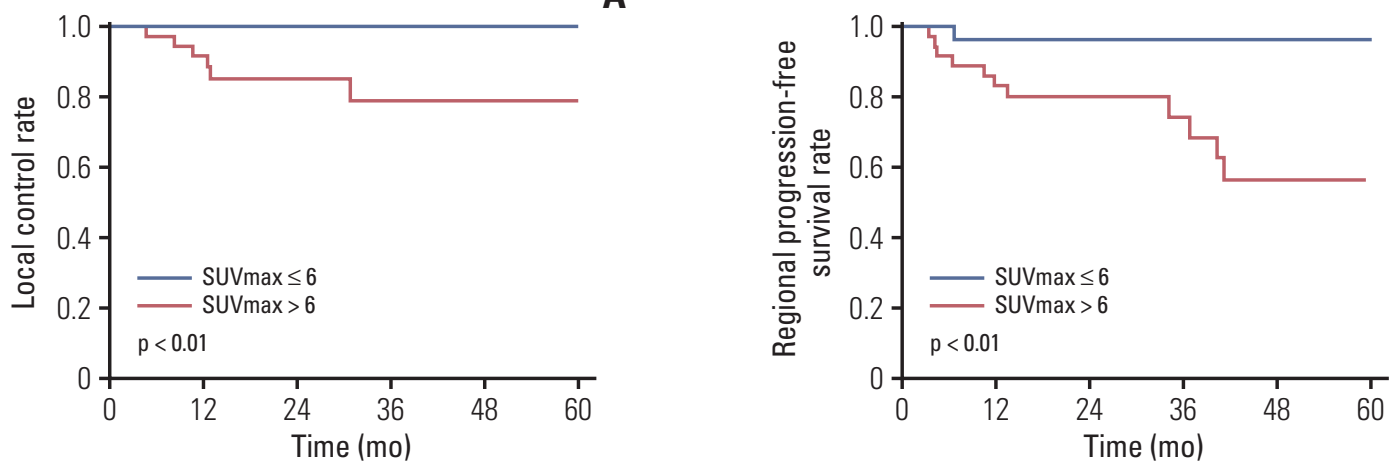

B

\section{C}
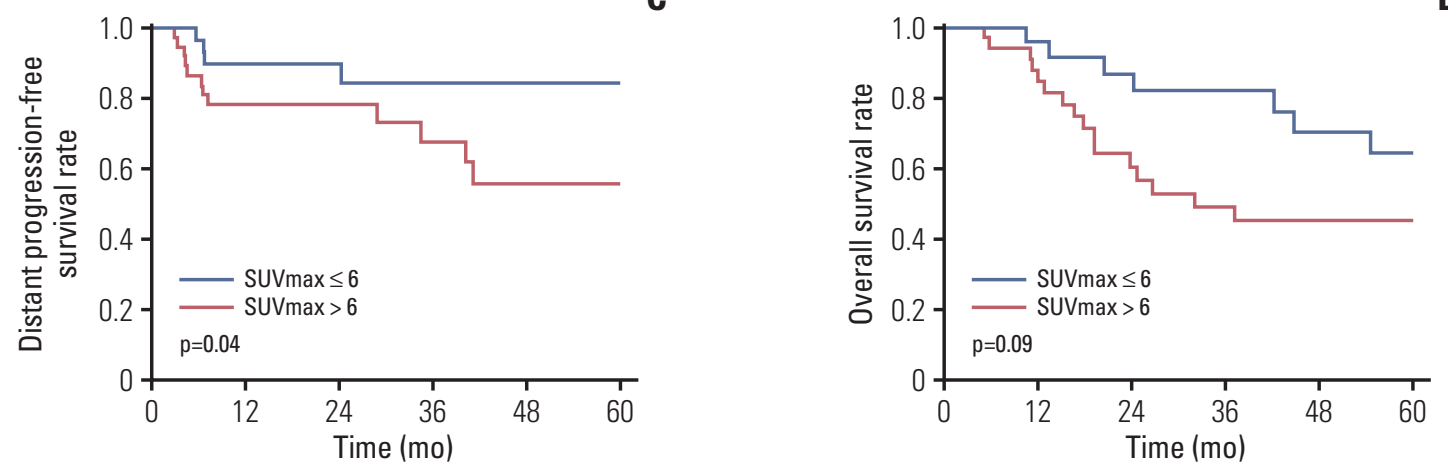

Fig. 3. Comparison of survival curves regarding pretreatment maximal standardized uptake value (SUVmax) level. (A) Local control rate. (B) Regional progression-free survival rate. (C) Distant progression-free survival rate. (D) Overall survival rate.

Above all, it demonstrates tumor metabolism and biology by measuring the FDG uptake of tumor cells. Based on this point of view, several studies have tried to predict the treatment response and recurrence with SUVmax of post-treatment PET-CT. However, post-radiation changes induce increased FDG uptake, which makes it extremely difficult to distinguish the radiation-induced change from local recurrence after SABR [14]. After serial PET-CTs obtained at 2, 26, and 52 weeks post-SABR, Henderson et al. [15] concluded that a substantial fraction of lesions showed a moderately elevated SUVmax at 1 year after SABR without local recurrence on further follow-up. Wiegman et al. [16] also conducted a serial PET, including 12 weeks post-SABR. They also were skeptical of the predictive value of post-treatment SUVmax. Therefore, it would be useful if the pretreatment SUVmax could predict treatment response and prognosis of the disease, since most patients obtain a baseline PET-CT at staging and the value is less likely to be obscured by other factors.

Actually, there are several studies that examined the potency of the pretreatment SUVmax as a predictive factor in NSCLC. Some reported that the pretreatment SUVmax failed as a predictor in stage I NSCLC treated with SABR. Burdick et al. [17] conducted a study on 72 patients treated with stereotactic body radiotherapy (SBRT) for T1-2N0M0 NSCLC to assess the predictive value of pretreatment PET SUVmax. However, the results were statistically insignificant. In their study, 32\% of the patients were not histologically proven, and this proportion can statistically influence the results. Also, the paper lacks on the information on SUVmax cut-off value. They employed SUVmax 5 as a cut-off, but the evidence supporting the numerical value is not clarified. Moreover, the median follow-up time was 16.9 months, which is shorter than our study. Similarly, another study by Miller et al. [18] argues on the lack of evidence on PET SUVmax as a predictor after SBRT for early-stage NSCLC. They delivered relatively low radiation dose of median $50 \mathrm{~Gy}$ in five fractions (more than $70 \%$ were treated with this scheme), which yields $100 \mathrm{BED}_{10}$. In our study, median $\mathrm{BED}_{10}$ delivered to a tumor was $116 \mathrm{~Gy}$ and this is a critical factor that affects treatment outcomes and our treatment outcomes are actually superior. Furthermore, their study focused only on distant failure rate, which is another difference with ours. Our study evaluated all survival outcomes. High SUVmax 


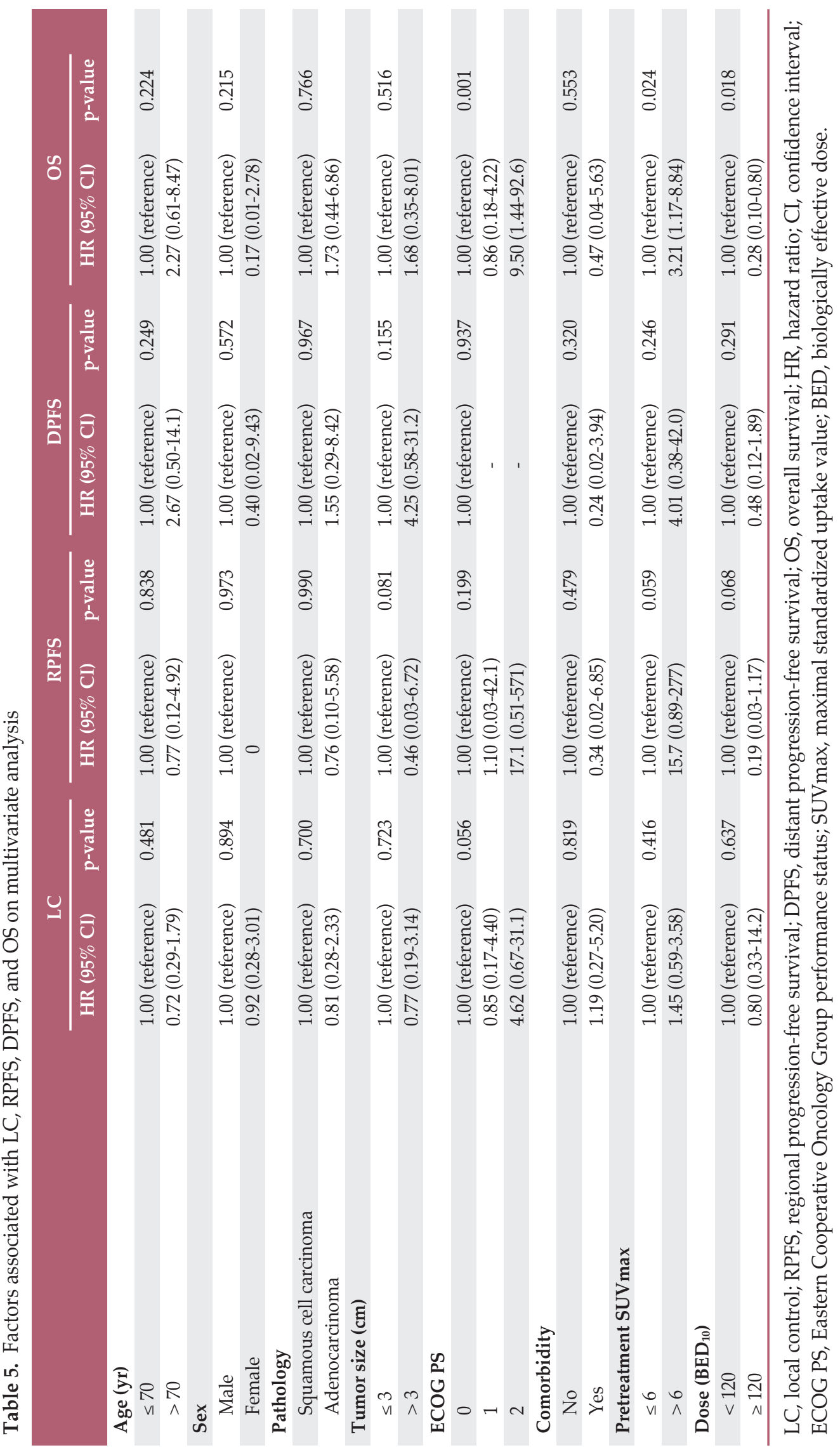


Table 6. Characteristic difference regarding SUVmax

\begin{tabular}{|c|c|c|c|}
\hline & SUVmax $\leq 6$ & SUVmax $>6$ & p-value \\
\hline \multicolumn{4}{|l|}{ Age $(\mathrm{yr})$} \\
\hline$\leq 70$ & $8(32.0)$ & $9(26.5)$ & 0.643 \\
\hline$>70$ & $17(68.0)$ & $25(73.5)$ & \\
\hline \multicolumn{4}{|l|}{ Sex } \\
\hline Male & $18(72.0)$ & $30(88.2)$ & 0.114 \\
\hline Female & $7(28.0)$ & $4(11.8)$ & \\
\hline \multicolumn{4}{|l|}{ ECOG performance status } \\
\hline 0 & $3(12.0)$ & $4(11.8)$ & 0.835 \\
\hline 1 & $19(76.0)$ & $24(70.6)$ & \\
\hline 2 & $3(12.0)$ & $6(17.6)$ & \\
\hline \multicolumn{4}{|l|}{ Comorbidity } \\
\hline No & $2(8.0)$ & $1(2.9)$ & 0.382 \\
\hline Yes & $23(92.0)$ & $33(97.1)$ & \\
\hline \multicolumn{4}{|l|}{ Pathology } \\
\hline Squamous cell carcinoma & $6(24.0)$ & $15(44.1)$ & 0.436 \\
\hline Adenocarcinoma & $15(60.0)$ & $14(41.2)$ & \\
\hline \multicolumn{4}{|l|}{ Tumor size (cm) } \\
\hline$\leq 3$ & $23(92.0)$ & $24(70.6)$ & 0.043 \\
\hline$>3$ & $2(8.0)$ & $10(29.4)$ & \\
\hline \multicolumn{4}{|l|}{ Pretreatment CEA $(\mathrm{ng} / \mathrm{mL})$} \\
\hline$<3$ & $11(68.8)$ & $12(50.0)$ & 0.240 \\
\hline$\geq 3$ & $5(31.3)$ & $12(50.0)$ & \\
\hline \multicolumn{4}{|l|}{ SABR dose $\left(\mathrm{BED}_{10}\right)$} \\
\hline$<120$ & $12(48.0)$ & $16(47.1)$ & 0.943 \\
\hline$\geq 120$ & $13(52.0)$ & $18(52.9)$ & \\
\hline
\end{tabular}

SUVmax, maximal standardized uptake value; ECOG, Eastern Cooperative Oncology Group; CEA, carcinoembrionic antigen; $\mathrm{SABR}$, stereotactic ablative body radiotherapy; $\mathrm{BED}$, biologically effective dose.

of tumor may influence on local failure and also distant metastasis.

Sasaki et al. [19] reported SUVmax as the most powerful factor in predicting disease-free survival and overall survival after curative surgery or radiotherapy in stage I-IIIB NSCLC. Zhang et al. [20] reported that the baseline SUVmax was predictive of local control after SABR in univariate analysis. A retrospective study by Park et al. [21] demonstrated SUVmax as a predictive value for progression-free survival after SABR in lung cancer and that SUVmax higher than 5.1 was correlated with progression. Moreover, in a recent meta-analysis that included studies reporting the hazard ratio of the pretreatment SUVmax for various outcome measures, higher SUVmax was associated with poorer local control (hazard ratio [HR], 1.13; 95\% CI, 1.06 to 1.21; $\mathrm{p}=0.0003$ ), more distant metastasis (HR, 1.09; 95\% CI, 1.03 to $1.16 ; \mathrm{p}=0.005$ ), and shorter overall survival (HR, 1.10; 95\% CI, 1.05 to 1.15 ; $p<0.001$ ) [22]. One of the earlier studies conducted by Yamamoto et al. [23] suggested the value of the pretreatment SUVmax in predicting local control after SABR, but this study also included advanced-stage NSCLC patients. Several groups that included only stage I NSCLC patients showed a strong correlation between the baseline SUVmax and progression-free survival $[24,25]$. A study with the largest cohort with only stage I NSCLC demonstrated SUVmax as a predictor for survival [26]. The 2-year rates of these outcome measures for low SUVmax versus high SUV-max groups in the previous studies of NSCLC treated with SABR are summarized in chronological order (Table 7).

Our treatment results were comparable to the previous reports, showing a statistically significant association of baseline SUVmax with local control, regional, and distant progression-free survival. The strength of this study is relatively longer follow-up duration (median, 32 months) and sufficient dose (median $\mathrm{BED}_{10}, 116 \mathrm{~Gy}$ ) delivered to histologically proven cases $(88.2 \%)$. Moreover, SUVmax cut-off value was determined with statistically reasonable methods.

Defining the optimal SUVmax to predict treatment outcomes is another challenging issue. In previously reported studies, the proposed optimal value of SUVmax was very 


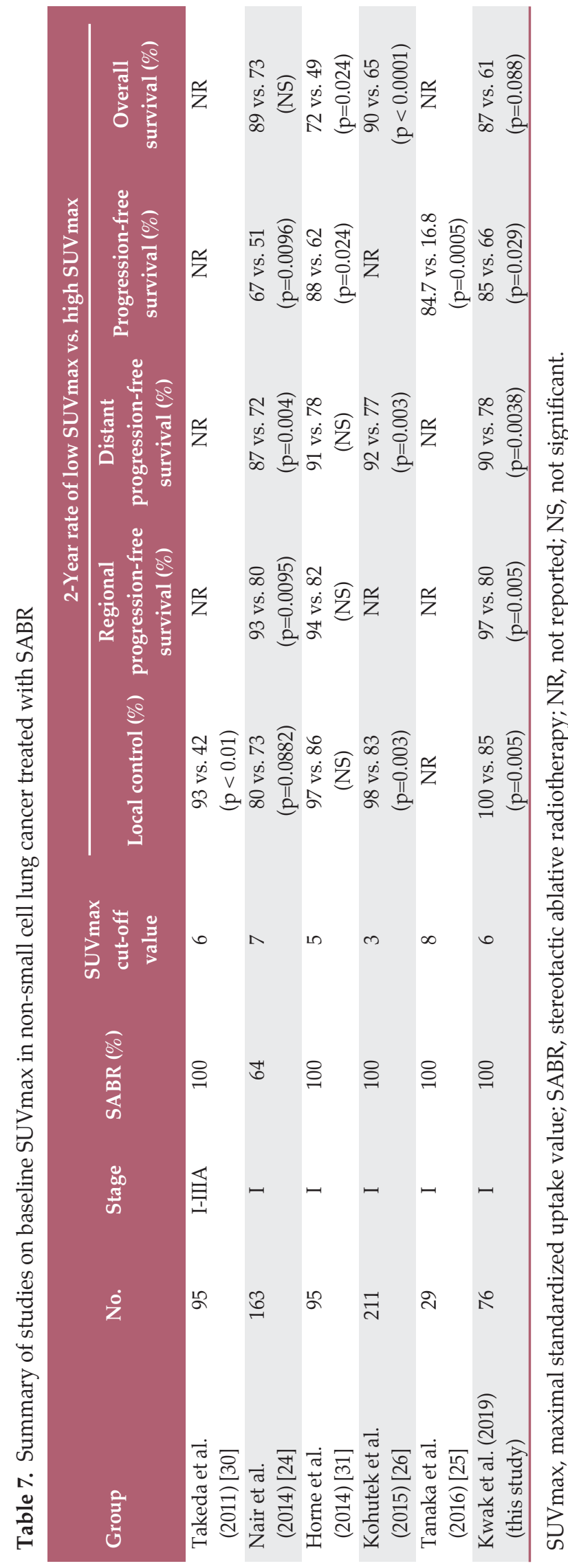

variable, ranging between 2.5 and 15 [6]. The median baseline SUVmax suggested in previous studies including our study was $6[17,24-31]$. Our cut-off value was determined by Cox proportional hazards model and ROC curve, which both indicated 6 as the most reasonable value. Morever, the Kaplan-Meier survival curves with log-rank test showed statistical difference between SUVmax $\leq 6$ and $>6$. Therefore, we can speculate on tumors with high SUVmax values greater than 6 may have poorer outcome after SABR.

The major limitation of this study is the limited number of patients, since surgery is the first treatment option that is most commonly proposed to early-staged patients. The lack of association with local control and distant progression-free survival may have been due to the small number of events. Heterogeneity in dose prescription and treatment modality is another limiting factor. Heterogeneous dose prescription may have derived from the retrospective nature of this study. When deciding upon radiation dose and fractionation scheme, we ponder on several characteristics such as tumor size, tumor location, underlying lung condition, age, performance status, pulmonary function, and combined comorbidities. Therefore, a kind of tailored treatment on each case is performed. Main factors for deciding dose scheme were tumor location (central vs. peripheral, or proximity to chest wall) and tumor size. Although dose profiles are somewhat heterogeneous, our tailored treatment might have contributed toward the excellent results.

This study concentrated on the prognostic value of baseline SUVmax in the treatment of early-stage NSCLC with SABR. We drew on a conclusion that tumors with SUVmax exceeding 6 have worse prognosis with high possibility of treatment failure and poor overall survival. On the basis of this finding, we suggest that more careful pretreatment staging work-up such as mediastinal and hilar lymph node sampling through EBUS and also more aggressive treatment, if feasible, with tumors with high pretreatment SUVmax. This aggressive treatment could be intensified radiation dose to the tumor, adjuvant systemic strategy including target agent or combining immunologic agent with SABR. However, since the evidence supporting these aforementioned approaches are not yet settled. Additional prospective trials with more inclusion of patients and more uniform treatment should be executed to propose on more specific treatment strategy according to the baseline SUVmax.

The current study implies the prognostic importance of the pre-SABR SUVmax and its potential role in categorizing patients to high and low risk. In conclusion, high baseline SUVmax above 6 may predict disease progression in stage I NSCLC after SABR. A prospective trial with further inclusion of patients and more uniform treatment may clearly elucidate the association between the pretreatment SUVmax and outcome after SABR. Likewise, the results will guide on the 
radiation dose prescription according to baseline SUVmax. Furthermore, we can decide upon adjuvant systemic strategy such as target agents or immune modulation or even regional radiation therapy in patients predicted with high risks.

\section{Conflicts of Interest}

Conflict of interest relevant to this article was not reported.

\section{References}

1. Senan S, Paul MA, Lagerwaard FJ. Treatment of early-stage lung cancer detected by screening: surgery or stereotactic ablative radiotherapy? Lancet Oncol. 2013;14:e270-4.

2. Nguyen NP, Garland L, Welsh J, Hamilton R, Cohen D, VinhHung V. Can stereotactic fractionated radiation therapy become the standard of care for early stage non-small cell lung carcinoma. Cancer Treat Rev. 2008;34:719-27.

3. Huang K, Palma DA; IASLC Advanced Radiation Technology Committee. Follow-up of patients after stereotactic radiation for lung cancer: a primer for the nonradiation oncologist. J Thorac Oncol. 2015;10:412-9.

4. Goldstraw P, Chansky K, Crowley J, Rami-Porta R, Asamura $\mathrm{H}$, Eberhardt WE, et al. The IASLC Lung Cancer Staging Project: proposals for revision of the TNM stage groupings in the forthcoming (eighth) edition of the TNM classification for lung cancer. J Thorac Oncol. 2016;11:39-51.

5. Berghmans T, Dusart M, Paesmans M, Hossein-Foucher C, Buvat I, Castaigne C, et al. Primary tumor standardized uptake value (SUVmax) measured on fluorodeoxyglucose positron emission tomography (FDG-PET) is of prognostic value for survival in non-small cell lung cancer (NSCLC): a systematic review and meta-analysis (MA) by the European Lung Cancer Working Party for the IASLC Lung Cancer Staging Project. J Thorac Oncol. 2008;3:6-12.

6. Paesmans M, Berghmans T, Dusart M, Garcia C, HosseinFoucher C, Lafitte JJ, et al. Primary tumor standardized uptake value measured on fluorodeoxyglucose positron emission tomography is of prognostic value for survival in non-small cell lung cancer: update of a systematic review and metaanalysis by the European Lung Cancer Working Party for the International Association for the Study of Lung Cancer Staging Project. J Thorac Oncol. 2010;5:612-9.

7. Goldstraw P. The 7th edition of the TNM classification for lung cancer: proposals from the IASLC staging project. Eur J Cancer Suppl. 2007;5:15-22.

8. Lee DS, Kim YS, Yoo IR, Kang YN, Kim SJ, Oh JK, et al. Longterm clinical experience of high-dose ablative lung radiotherapy: high pre-treatment [18F]fluorodeoxyglucose-positron emission tomography maximal standardized uptake value of the primary tumor adversely affects treatment outcome. Lung Cancer. 2013;80:172-8.

9. Graziano SL. Non-small cell lung cancer: clinical value of new biological predictors. Lung Cancer. 1997;17 Suppl 1:S37-58.

10. Jeremic B, Milicic B, Dagovic A, Acimovic L, Milisavljevic S. Pretreatment prognostic factors in patients with early-stage
(I/II) non-small-cell lung cancer treated with hyperfractionated radiation therapy alone. Int J Radiat Oncol Biol Phys. 2006;65:1112-9.

11. Trape J, Buxo J, Perez de Olaguer J, Vidal C. Tumor markers as prognostic factors in treated non-small cell lung cancer. Anticancer Res. 2003;23:4277-81.

12. Niklinski J, Niklinska W, Laudanski J, Chyczewska E, Chyczewski L. Prognostic molecular markers in non-small cell lung cancer. Lung Cancer. 2001;34 Suppl 2:S53-8.

13. Li Q, Kim J, Balagurunathan Y, Qi J, Liu Y, Latifi K, et al. CT imaging features associated with recurrence in non-small cell lung cancer patients after stereotactic body radiotherapy. Radiat Oncol. 2017;12:158.

14. Matsuo Y, Nakamoto Y, Nagata Y, Shibuya K, Takayama K, Norihisa $Y$, et al. Characterization of FDG-PET images after stereotactic body radiation therapy for lung cancer. Radiother Oncol. 2010;97:200-4.

15. Henderson MA, Hoopes DJ, Fletcher JW, Lin PF, Tann M, Yiannoutsos CT, et al. A pilot trial of serial 18F-fluorodeoxyglucose positron emission tomography in patients with medically inoperable stage I non-small-cell lung cancer treated with hypofractionated stereotactic body radiotherapy. Int J Radiat Oncol Biol Phys. 2010;76:789-95.

16. Wiegman EM, Pruim J, Ubbels JF, Groen HJ, Langendijk JA, Widder J. 18F-FDG PET during stereotactic body radiotherapy for stage I lung tumours cannot predict outcome: a pilot study. Eur J Nucl Med Mol Imaging. 2011;38:1059-63.

17. Burdick MJ, Stephans KL, Reddy CA, Djemil T, Srinivas SM, Videtic GM. Maximum standardized uptake value from staging FDG-PET/CT does not predict treatment outcome for early-stage non-small-cell lung cancer treated with stereotactic body radiotherapy. Int J Radiat Oncol Biol Phys. 2010;78:10339.

18. Miller CJ, Martin B, Stang K, Hutten R, Alite F, Small C, et al. Predictors of distant failure after stereotactic body radiation therapy for stages I to IIA non-small-cell lung cancer. Clin Lung Cancer. 2019;20:37-42.

19. Sasaki R, Komaki R, Macapinlac H, Erasmus J, Allen P, Forster $\mathrm{K}$, et al. [18F]fluorodeoxyglucose uptake by positron emission tomography predicts outcome of non-small-cell lung cancer. J Clin Oncol. 2005;23:1136-43.

20. Zhang X, Liu H, Balter P, Allen PK, Komaki R, Pan T, et al. Positron emission tomography for assessing local failure after stereotactic body radiotherapy for non-small-cell lung cancer. Int J Radiat Oncol Biol Phys. 2012;83:1558-65. 
21. Park J, Choi Y, Ahn KJ, Park SK, Cho H, Lee JY. Maximum standardized uptake value at pre-treatment PET in estimating lung cancer progression after stereotactic body radiotherapy. Radiat Oncol J. 2019;37:30-6.

22. Dong M, Liu J, Sun X, Xing L. Prognositc significance of SUVmax on pretreatment (18) F-FDG PET/CT in early-stage nonsmall cell lung cancer treated with stereotactic body radiotherapy: A meta-analysis. J Med Imaging Radiat Oncol. 2017; 61:652-9.

23. Yamamoto T, Kadoya N, Shirata Y, Kaneta T, Koto M, Umezawa R, et al. Formula corrected maximal standardized uptake value in FDG-PET for partial volume effect and motion artifact is not a prognostic factor in stage I non-small cell lung cancer treated with stereotactic body radiotherapy. Ann Nucl Med. 2015;29:666-73.

24. Nair VJ, MacRae R, Sirisegaram A, Pantarotto JR. Pretreatment [18F]-fluoro-2-deoxy-glucose positron emission tomography maximum standardized uptake value as predictor of distant metastasis in early-stage non-small cell lung cancer treated with definitive radiation therapy: rethinking the role of positron emission tomography in personalizing treatment based on risk status. Int J Radiat Oncol Biol Phys. 2014;88:312-8.

25. Tanaka H, Hayashi S, Hoshi H. Pretreatment maximum standardized uptake value on 18F-fluorodeoxyglucose positron emission tomography is a predictor of outcome for stage I nonsmall cell lung cancer after stereotactic body radiotherapy. Asia Pac J Clin Oncol. 2016;12:e113-7.
26. Kohutek ZA, Wu AJ, Zhang Z, Foster A, Din SU, Yorke ED, et al. FDG-PET maximum standardized uptake value is prognostic for recurrence and survival after stereotactic body radiotherapy for non-small cell lung cancer. Lung Cancer. 2015;89: $115-20$.

27. Clarke K, Taremi M, Dahele M, Freeman M, Fung S, Franks K, et al. Stereotactic body radiotherapy (SBRT) for non-small cell lung cancer (NSCLC): is FDG-PET a predictor of outcome? Radiother Oncol. 2012;104:62-6.

28. Chang JY, Liu H, Balter P, Komaki R, Liao Z, Welsh J, et al. Clinical outcome and predictors of survival and pneumonitis after stereotactic ablative radiotherapy for stage I non-small cell lung cancer. Radiat Oncol. 2012;7:152.

29. Vu CC, Matthews R, Kim B, Franceschi D, Bilfinger TV, Moore WH. Prognostic value of metabolic tumor volume and total lesion glycolysis from (1)(8)F-FDG PET / CT in patients undergoing stereotactic body radiation therapy for stage I nonsmall-cell lung cancer. Nucl Med Commun. 2013;34:959-63.

30. Takeda A, Yokosuka N, Ohashi T, Kunieda E, Fujii H, Aoki Y, et al. The maximum standardized uptake value (SUVmax) on FDG-PET is a strong predictor of local recurrence for localized non-small-cell lung cancer after stereotactic body radiotherapy (SBRT). Radiother Oncol. 2011;101:291-7.

31. Horne ZD, Clump DA, Vargo JA, Shah S, Beriwal S, Burton $S A$, et al. Pretreatment SUVmax predicts progression-free survival in early-stage non-small cell lung cancer treated with stereotactic body radiation therapy. Radiat Oncol. 2014;9:41. 